\title{
Linking dynamical perceptual decisions at different levels of description in motion pattern formation: Psychophysics
}

\author{
HOWARD S. HOCK \\ Florida Atlantic University, Boca Raton, Florida \\ and \\ ANNEMIE PLOEGER \\ University of Amsterdam, Amsterdam, The Netherlands
}

\begin{abstract}
The relationship between local-level motion detection and higher level pattern-forming mechanisms was investigated with the motion quartet, a bistable stimulus for which either horizontal or vertical motion patterns are perceived. Local-level perturbations in luminance contrast affected the stability of the perceived patterns and, thereby, the size of the pattern-level hysteresis obtained by gradually changing the motion quartet's aspect ratio. Briefly eliminating luminance contrast (so nonmotion was perceived during the perturbation) eliminated pattern-level hysteresis, and briefly increasing luminance contrast (so motion was perceived during the perturbation) increased pattern-level hysteresis. Partially reducing luminance contrast resulted in bistability during the perturbation; pattern-level hysteresis was maintained when motion was perceived, and eliminated when nonmotion was perceived. The results were attributed to local motion/nonmotion perceptual decisions in area V1 affecting the magnitude of the activation feeding forward to motion detectors in area MT, where the stability of pattern-level perceptual decisions is determined by activation-dependent, future-shaping interactions that inhibit soon-to-be-stimulated detectors responsive to competing motion directions.
\end{abstract}

An ongoing issue in our understanding of visual pattern formation concerns the relationship between different levels of description for the pattern. Although the traditional idea is that the relationship is hierarchical, with larger units built from smaller ones (see, e.g., Biederman, 1987; Cutting, 1986), it also has been argued that processing proceeds in the reverse, top-down direction (Hochstein \& Ahissar, 2002; Sanocki, 1993). Upstream areas of the brain have larger receptive fields and respond to more abstract, global properties than do downstream areas (Maunsell \& Newsome, 1987; Vogels \& Orban, 1996), so different levels of description are likely to have their counterparts in different levels of neural processing.

The present research investigates the feedforward flow of activation from the detection of local element motions to the formation of simple global motion patterns. Psychophysical results in this article and computational simulations of these results in the accompanying article (Nichols, Hock, \& Schöner, 2006) indicate that (1) pattern-level hysteresis depends on activation-dependent detector interactions and, therefore, on the magnitude of the activation that feeds forward from local to pattern levels, and

We thank Gregor Schöner and David Nichols for their careful reading of the manuscript and valuable suggestions. Correspondence relating to this article may be sent to H. S. Hock, Department of Psychology, Florida Atlantic University, Boca Raton, FL 33431 (e-mail: hockhs@fau.edu).
(2) local-level dynamical decisions that result in motion or nonmotion being perceived (i.e., local bistability) affect pattern-level dynamical decisions by feeding forward different magnitudes of activation for the same stimulus. Dynamical decisions occur when the activation levels of stimulated detectors are attracted to and maintained near stable, fixed-point values (Hock, Schöner, \& Giese, 2003), where each fixed point represents the joint activation state of all detectors relevant to the percept. It is assumed that the percept is embodied in those detectors whose activation is stabilized at values above the threshold level required for perception.

Hock, Kogan, and Espinoza (1997) and Hock, Nichols, and Espinoza (2004) have provided psychophysical evidence that detectors responsive to motion-independent spatial information compete with motion detectors in determining whether motion or nonmotion is perceived. They observed spontaneous switching between nonmotion and motion percepts, motion/nonmotion hysteresis, and the independent adaptation of perceived nonmotion and perceived motion. Our account therefore begins in area $\mathrm{V} 1$, where in addition to directionally selective motion detectors (Hubel \& Wiesel, 1979) there are detectors responsive to motion-independent spatial information (Movshon, Thompson, \& Tolhurst, 1978). Detectors in area MT are predominantly motion sensitive (Maunsell \& Van Essen, 1983), so V1 is the more likely of the two sites for motion/nonmotion decisions. Directionally selec- 
tive motion detectors in V1 transmit their activation to MT motion detectors with corresponding directional selectivity (Movshon \& Newsome, 1996), so differences in local stimulus strength as well as local motion/nonmotion decisions in V1 can affect MT activation.

V1 and MT differ in that only in MT do systematic inhibitory interactions occur among detectors with different directional selectivity (see, e.g., Snowden, Treue, Erickson, \& Andersen, 1991; Thiele, Dobkins, \& Albright, 2000). In addition, computational simulations for random cinematograms (Williams, Phillips, \& Sekuler, 1986), plaids (Wilson \& Kim, 1994), and the motion quartet (see, e.g., Bartsch \& van Hemmen, 1997; Carmesin \& Arndt, 1996; Hock et al., 2003) indicate that motion pattern formation depends on recursive inhibitory interactions among detectors with different directional selectivity. MT therefore is a likely site for pattern-level dynamical decisions (see, e.g., Stoner \& Albright, 1992).

The stimulus we've studied is the motion quartet. In its original version (Figure 1A), two elements are presented in opposite corners of an imaginary rectangle, then shifted to the other corners, back and forth (Hock, Kelso, \& Schöner, 1993; Hoeth, 1968; Kruse, Stadler, \& Wehner, 1986; Ramachandran \& Anstis, 1985; von Schiller, 1933). Whether horizontal or vertical motion is perceived depends on the aspect ratio (the vertical divided by the horizontal interelement distance), because shorter motion paths produce more motion detector activation than do longer motion paths (Gilroy, Hock, \& Ploeger, 2001). This provides the basis for the "nearest neighbor" solution to emerge from activation-dependent inhibitory interactions among simultaneously stimulated horizontal and vertical detectors; that is, detectors that are more activated (shorter motion paths) inhibit their less activated competitors (longer motion paths) more than the less activated detectors inhibit them. Hock et al. (2003) called these "patternforming" interactions (Figure 1C).

The quartets in the present study had simultaneously visible elements in all four corners (Figure 1B). Elements in opposite corners had one luminance value, and those in the other corners had a different value. Motion can be perceived when the luminance values are exchanged, depending on the background-relative luminance change, or BRLC (Hock et al., 1997). The BRLC value is the frameto-frame change in luminance divided by the difference between the average luminance and the luminance of the background. Its value in the present study, 0.8, was sufficiently large for motion to almost always be perceived.

The stability of pattern-level dynamical decisions was observed by measuring hysteresis. This entailed gradually increasing or gradually decreasing the aspect ratio of the motion quartet and determining when switches occurred between the initially established and the competing motion pattern. In a series of computational simulations, Hock et al. (2003) showed that as the aspect ratio is changed, pattern-level perceptual decisions are determined by interactive influences of currently activated detectors on detectors with orthogonal directional selectivity that will be stimulated in the immediate future. For example, when horizontal motion patterns are perceived (the activation of vertical motion is suppressed), and both horizontal and vertical directions are about to be stimulated, the to-bestimulated vertical motion detectors will be inhibited by the currently activated horizontal motion detectors, predisposing the continued perception of horizontal motion. When these "future-shaping" interactions (Figure 1D) were removed from the simulations, continual switching between the horizontal and vertical motion patterns was signified (stability was lost), and hysteresis effects were not obtained, contrary to what is perceived in reality. Future-shaping interactions thus result in state dependence, a predisposition to the immediately preceding perceptual/activation state that leads to the initially established pattern persisting in perception, even as aspect ratios are changed to values favoring the formation of the alternative pattern (Hock et al., 1993).

The key to the experiments here and the computational simulations in the accompanying article (Nichols et al., 2006) is the activation dependence of future-shaping interactions. When the activation transmitted from the local to the pattern level is relatively low, future-shaping interactions are expected to be weak; horizontal motion can be perceived, but the vertical motion detectors that will be stimulated in the immediate future would be only weakly inhibited. There would be relatively little predisposition to the continued perception of horizontal motion, so relatively little pattern-level hysteresis would be expected. However, when the activation transmitted from the local to the pattern level is increased, the activation embodying perceived horizontal motion patterns will increase, and stronger future-shaping interactions would inhibit vertical motion detectors that will be stimulated in the immediate future, providing a strong predisposition to the continued perception of horizontal motion (and, thus, increased pattern-level hysteresis).

The activation dependence of future-shaping interactions was probed with brief perturbations in BRLC near the end of trials with increasing and decreasing aspect ratios. The perturbations were nonspecific with respect to the local activation of horizontal and vertical motion detectors, so their effect on pattern-level decisions was expected to depend on the level of activation feeding forward from V1 to MT, and thus on the strength of activation-dependent, future-shaping interactions among MT detectors. Because the predicted effect of the perturbation was to change the strength of future-shaping interactions, changes in pattern stability (and, thus, hysteresis) were expected at all aspect ratios for which there was a perturbation. In Experiment 1, the BRLC decreased to 0 during the perturbation, suspending the transmission of activation to MT. In Experiment 2, it increased to 2.0 during the perturbation, increasing the activation transmitted to MT. In Experiment 3, it decreased to 0.4 , creating local motion/nonmotion bistability, and thereby transmitting discretely different levels of activation to MT for the same stimulus.

\section{METHOD}

Pattern-level hysteresis was measured using the modified method of limits developed by Hock et al. (1993). In the modified method, 
Small Aspect Ratios Favor

Vertical Motion Correspondences

A

Frame 1

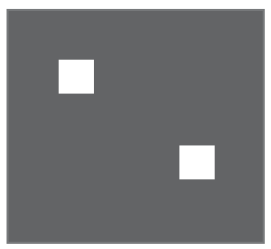

Frame 2

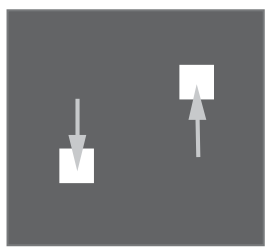

B

Frame 1
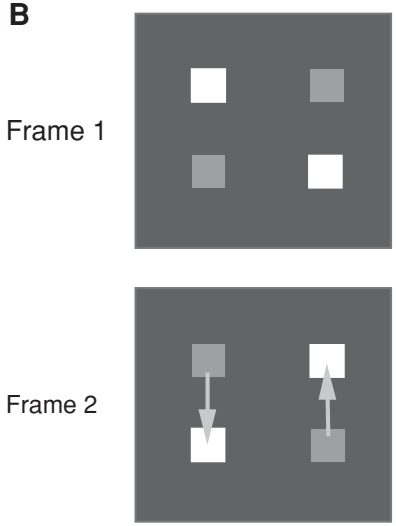

C Pattern-Forming Interactions

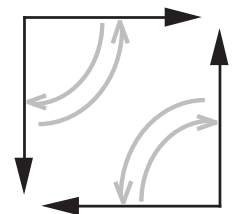

Large Aspect Ratios Favor Horizontal Motion Correspondences

Frame 1

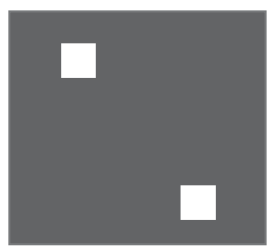

Frame 2

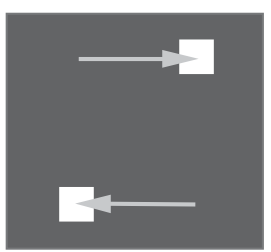

Frame 1

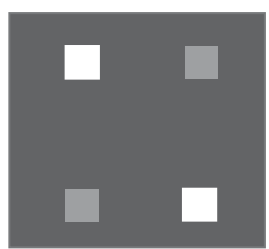

Frame 2

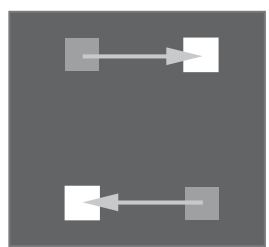

D Future-Shaping Interactions

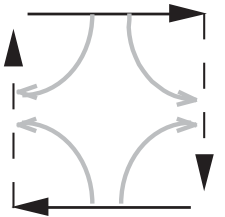

Figure 1. (A) Two-element version of the motion quartet, based on standard apparent motion; elements disappear from two locations and reappear at two other locations. (B) Four-element version of the motion quartet, based on generalized apparent motion (Hock et al., 1997); all four elements are simultaneously visible, and motion results from changes in luminance at each location. For both standard and generalized apparent motion, small aspect ratios favor the perception of vertical motion and large aspect ratios favor the perception of horizontal motion. (C) Pattern-forming interactions entail mutual inhibition between simultaneously stimulated motion directions. One or the other of the motion directions is perceived. (D) Future-shaping inhibitory interactions for sequentially stimulated motion directions. In this example, the continued perception of the horizontal motion perceived during frame $\boldsymbol{n}$ is predisposed by the inhibition of the vertical motion that will be stimulated during frame $n+1$.

observers withhold their response until the end of a trial, eliminating the midtrial responses that are the source of response hysteresis (the tendency to repeat the same response, despite changes in the percept) in the standard method of limits. In addition, separate ascending and descending trials vary with respect to how deeply a gradually increasing parameter (ascending trials) or a gradually decreasing parameter (descending trials) penetrates the bistable region within which two percepts are possible; that is, each trial has a different endpoint parameter value. The size of the hysteresis effect is determined by the endpoint parameter values that must be reached in order for 
switches to be observed. Larger hysteresis occurs when the trials for which there are switches have endpoint aspect ratios that are farther along in the ascending and descending series than would be expected from the stimulus-initiated activation of the alternative percepts. With this method, there is no need for participants to respond as soon as they experience a switch, and no need for the investigator to guess at the time required for a motor response to be generated in order to estimate when the switch actually was perceived.

\section{Participants}

In addition to the authors, the participants included 2 undergraduate and 3 graduate students at Florida Atlantic University who were naive with respect to the purpose of the experiments.

\section{Stimuli}

The motion quartet stimulus was composed of four small, brighterthan-background elements that were simultaneously presented in the center of a Macintosh II monitor within a darkened room. When viewed from a distance of $30 \mathrm{~cm}$ (maintained with a chinrest), each element subtended a visual angle of $8.1^{\prime}$. The elements were located in the corners of an imaginary rectangle whose aspect ratio, the vertical divided by the horizontal distance between the elements, varied between 0.5 and 2.0. The horizontal interelement distance always was $16.2^{\prime}$, so the different aspect ratios were created by varying the vertical interelement distance.

During each trial, the motion quartet was presented over a sequence of display cycles, each composed of two 195-msec frames. During one frame, one pair of diagonally opposite elements had one luminance value $\left(L_{1}\right)$, and the diagonally opposite pair had a different luminance value $\left(L_{2}\right)$. These luminance values were exchanged during the second frame, which constituted the display cycle, and the exchanges then continued for the entire trial. With the exception of frames during which there was a perturbation, the luminance change at each location, quantified as the BRLC, was $0.8\left(L_{1}=7.5 \mathrm{~cd} / \mathrm{m}^{2}\right.$, $L_{2}=5.9 \mathrm{~cd} / \mathrm{m}^{2}$, and $L=4.6 \mathrm{~cd} / \mathrm{m}^{2}$ for the background).

Both the starting and endpoint aspect ratios varied randomly from trial to trial. The starting aspect ratio was 0.5 for ascending trials (strongly favoring the perception of vertical motion) and 2.0 for descending trials (strongly favoring the perception of horizontal motion). The endpoint aspect ratio was either $0.75,1.0,1.25,1.5,1.75$, or 2.0 for ascending trials and $1.75,1.5,1.25,1.0,0.75$, or 0.5 for descending trials. During each trial, the starting aspect ratio was presented for three display cycles, then the aspect ratio was increased or decreased in steps of 0.25 (one step per two-frame display cycle) until the endpoint aspect ratio was reached. This aspect ratio was presented for an extra (third) frame to allow for recovery of motion perception following perturbations in BRLC, which were introduced during the preceding, next-to-last display cycle. The perturbations entailed repeating the next-to-last aspect ratio for two frames, but at luminance values that resulted in BRLC values other than 0.8 . (The average luminance of the elements was $6.7 \mathrm{~cd} / \mathrm{m}^{2}$ throughout each trial, regardless of whether or not there was a perturbation, and regardless of the size of the perturbation.)

The BRLC perturbation was 0 in Experiment 1. Its effect was spread over three frame changes. The BRLC for the frame change leading into the perturbation frames was 0.4 (halfway between 0 and 0.8 ), the BRLC for the frame change between the two perturbation frames was 0 , and the BRLC for the frame change leading out of the perturbation frames was 0.4 . Because the predicted effect of the perturbation at each frame change was to decrease the strength of future-shaping interactions, reductions in pattern stability (and thus, in hysteresis) were expected at all aspect ratios for which there was a perturbation. In Experiment 2, the BRLC perturbation was 2.0. The BRLC was 1.4 for the lead-in frame change (halfway between 0.8 and 2.0), 2.0 for the frame change within the perturbation, and 1.4 for the lead-out frame change. The predicted effect of the perturbation was to increase the strength of future-shaping interactions at each frame change, thereby increasing pattern stability (and thus, hysteresis) at all aspect ratios for which there was a perturbation. In Experiment 3, the BRLC perturbation was 0.4, again distributed over three frame changes $(0.6,0.4,0.6)$. It was predicted that the effect of this perturbation would depend on whether nonmotion was perceived as a result of any of the three frame changes, or whether motion was perceived throughout.

\section{Procedure}

At the end of each trial, the participants reported (by pressing keys on the computer keyboard) whether nonmotion was perceived at any time during the trial, and in addition, whether the perceived motion pattern changed at any time during the trial. A block of 192 orderrandomized trials was presented during each testing session. It was generated by the orthogonal combination of 12 trial types ( 6 ascending and 6 descending trials, each with a different endpoint aspect ratio), 2 perturbation conditions (half of the trials with a perturbation, half without), and 8 repetitions. The number of testing sessions for each participant varied across the three experiments.

\section{EXPERIMENT 1}

The perturbation in this experiment entailed a reduction of the BRLC from 0.8 to 0 during the next-to-last display cycle of both ascending and descending trials. Examples of trials with ascending aspect ratios are as follows.

Perturbation condition: 0.5 (6 frames), 0.75 ( 2 frames), 1.0 (2 frames), 1.25 (2 frames), 1.25 (2 frames with BRLC perturbation), 1.5 (3 frames);

No-perturbation condition: 0.5 (6 frames), 0.75 (2 frames), 1.0 ( 2 frames), 1.25 ( 2 frames), 1.5 (3 frames). ${ }^{1}$

There were four testing sessions for each of the 4 participants.

\section{Results}

The results for the 4 participants are presented in Figure 2. It can be seen that hysteresis was obtained in the noperturbation condition for all 4 participants. That is, the likelihood of perceptual switches between the horizontal and vertical motion patterns was different at intermediate endpoint aspect ratios, depending on whether the endpoint aspect ratio was reached through an ascending or descending trial. When the initially established motion pattern was vertical (ascending trials, shown by the open circles), the vertical pattern persisted despite endpoint aspect ratios being reached that favored the horizontal pattern. Conversely, when the initially established motion pattern was horizontal (descending trials, filled circles), the horizontal pattern persisted despite endpoint aspect ratios being reached that favored the vertical pattern.

In the perturbation condition, motion perception stopped while the BRLC was reduced to 0 during the next-to-last display cycle. When the BRLC value was restored to 0.8 , and motion perception resumed, this disruption was detrimental to hysteresis for all 4 participants. The hysteresis effect was reversed for A.P. and H.H., eliminated for J.E., and reduced for W.S. This finding was quantified by probit analysis. Cumulative normal distributions were fit to the empirical results for ascending and descending trials in order to find the $50 \%$ threshold for ascending trials (the endpoint aspect ratio for which there was a switch from 
No-Perturbation Condition
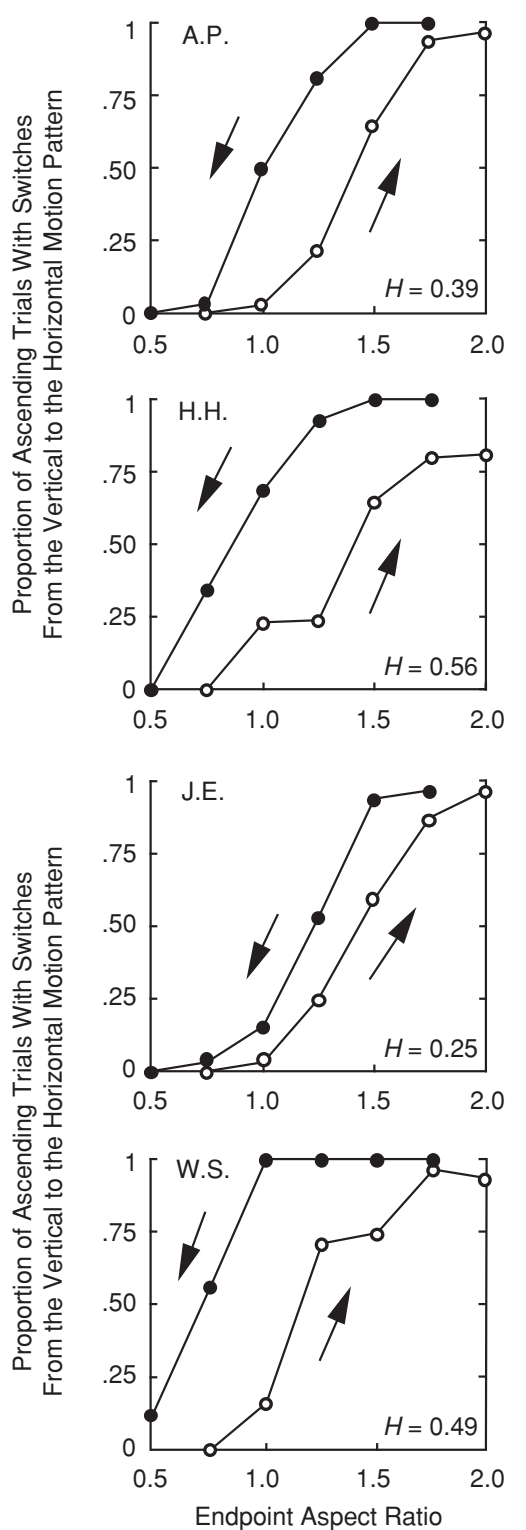

Perturbation $(B R L C=0)$ Condition
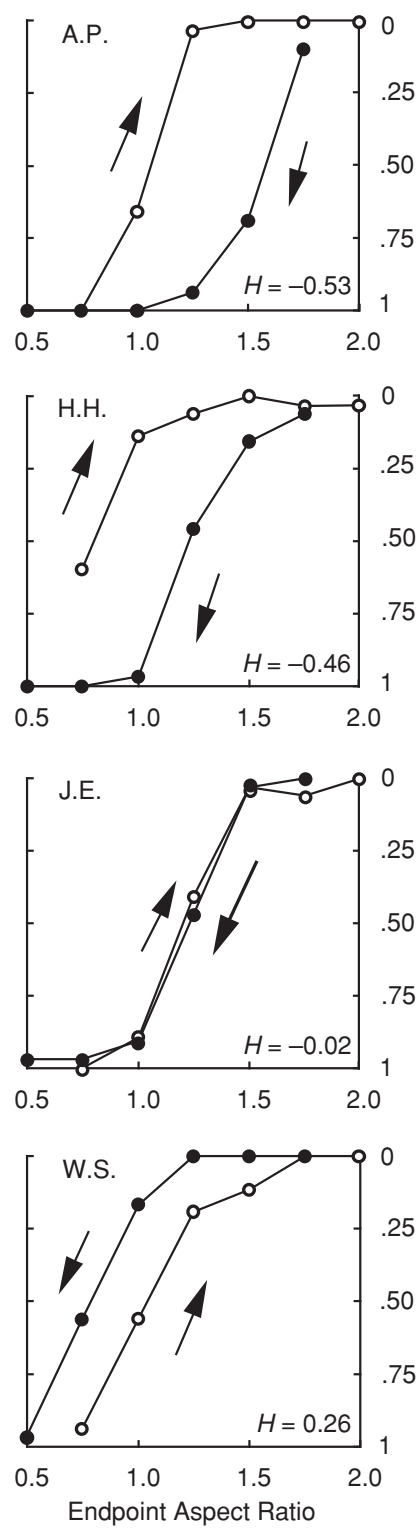

Figure 2. Experiment 1: Hysteresis effects for each participant in the no-perturbation and perturbation conditions (BRLC decreased from 0.8 to 0 during the next-to-last display cycle). The results indicate the proportions of trials in which change to an alternative percept occurred sometime during each trial. The axes on the left side of each graph indicate the results for ascending trials (i.e., those with increasing aspect ratios; open circles). The axes on the right side of each graph (note their inversion) indicate the results for descending trials (i.e., those with decreasing aspect ratios; filled circles). The size of each hysteresis effect, $H$, is indicated within each graph (see the text for the measurement procedure).

vertical to horizontal motion for half the trials) and the $50 \%$ threshold for descending trials (the endpoint aspect ratio for which there was a switch from horizontal to vertical motion for half the trials). The size of each hysteresis effect $(H)$ was the difference between the $50 \%$ thresholds for the ascending and descending trials. Values of $H$ are indicated in each panel of Figure 2.

\section{EXPERIMENT 2}

In Experiment 1, pattern-level hysteresis was curtailed by a perturbation that briefly suspended feedforward activation from the local motion to the pattern level. This was consistent with the hypothesis that there would be no future-shaping interactions to influence pattern formation 
No-Perturbation Condition
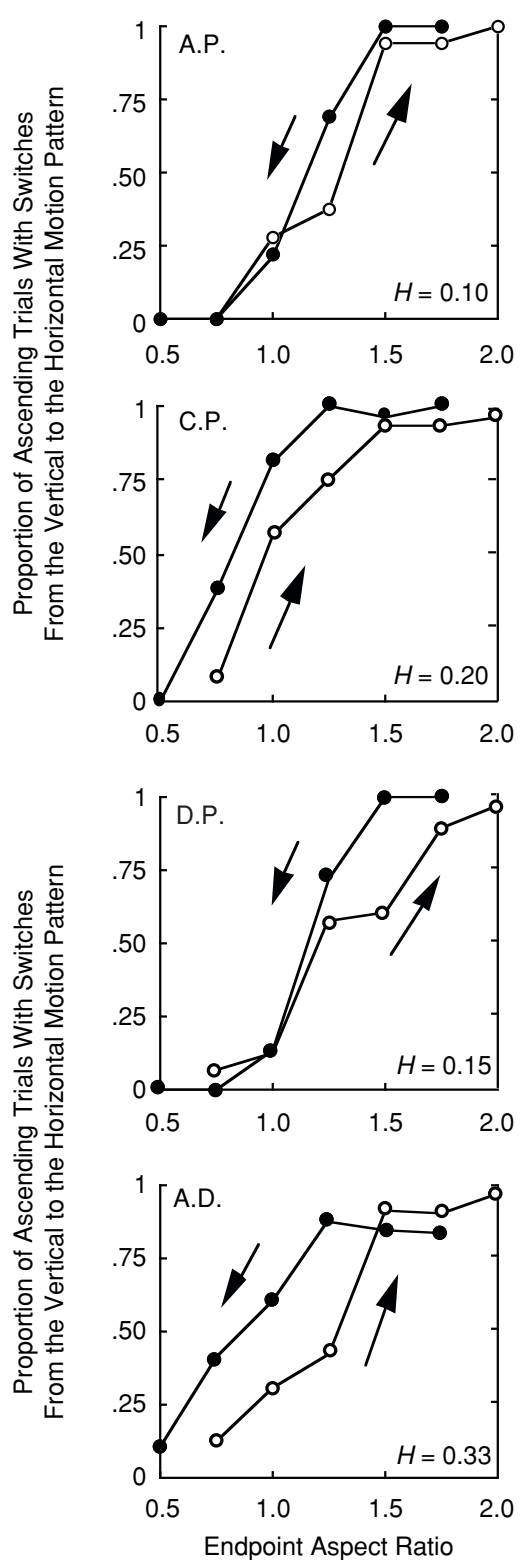

Perturbation $(B R L C=2.0)$ Condition
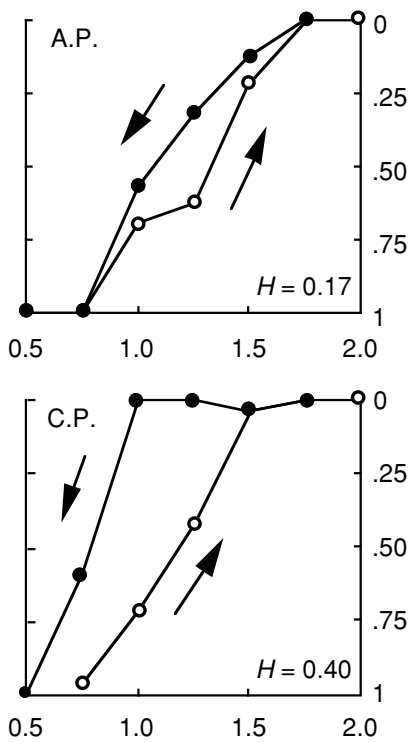

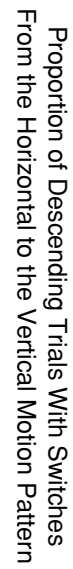
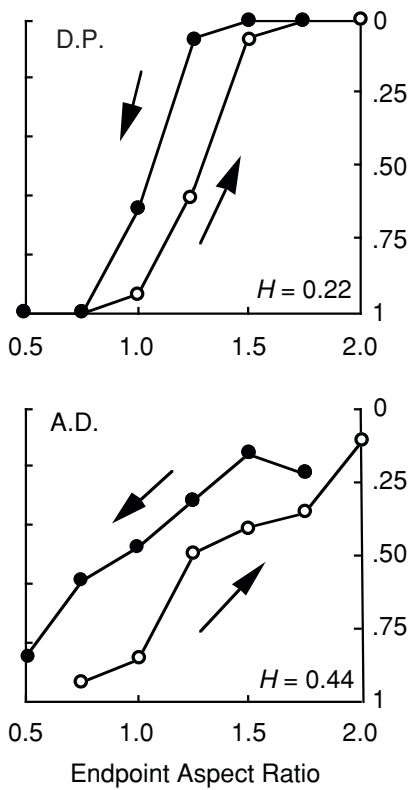

Figure 3. Experiment 2: Hysteresis effects for each participant in the no-perturbation and perturbation conditions (BRLC increased from 0.8 to 2.0 during the next-to-last display cycle). The axes on the left side of each graph indicate the results for ascending trials (those with increasing aspect ratios; open circles). The results indicate the proportions of trials in which change to an alternative percept occurred sometime during each trial. The axes on the right side of each graph (note their inversion) indicate the results for descending trials (those with decreasing aspect ratios; filled circles). The size of each hysteresis effect, $H$, is indicated within each graph (see the text for the measurement procedure).

when motion perception resumed after the perturbation. Following the same reasoning, it was anticipated that if the magnitude of feedforward activation was increased during the perturbation interval, the strength of future-shaping interactions would increase, increasing the size of the pattern-level hysteresis. To test this, the BRLC value was increased from 0.8 to 2.0 during the display cycle with the perturbation. The higher luminance value of each element during the perturbation was $8.8 \mathrm{~cd} / \mathrm{m}^{2}$, and the lower value was $4.6 \mathrm{~cd} / \mathrm{m}^{2}$. The latter luminance value was the same as 
the luminance of the background, so two of the four elements of the quartet were not visible during this perturbation. Examples of trials with ascending aspect ratios are:

Perturbation condition: 0.5 (6 frames), 0.75 ( 2 frames), 1.0 (2 frames), 1.25 (2 frames), 1.25 (2 frames with a BRLC perturbation), 1.5 (3 frames);

No-perturbation condition: 0.5 (6 frames), 0.75 (2 frames), 1.0 (2 frames), 1.25 (2 frames), 1.25 (2 frames without a perturbation), 1.5 (3 frames). ${ }^{2}$

There were four testing sessions for each of the 4 participants.

\section{Results}

Hysteresis effects again were obtained in the noperturbation condition for all 4 participants. However, in contrast with the reductions in hysteresis observed in the perturbation condition of Experiment 1, there were small to moderate increases in the size of the hysteresis in the perturbation condition for the 4 participants in this experiment (Figure 3). This was consistent with increased feedforward activation during the perturbation that strengthened future-shaping interactions prior to the BRLC returning to 0.8 . It is noteworthy that if the reduction in hysteresis by the BRLC $=0$ perturbation in Experiment 1 were due to the distraction of attention by the perturbation, a similar distraction-induced reduction in hysteresis would have occurred in this experiment, which would be the opposite of the observed results.

\section{EXPERIMENT 3}

The results of Experiments 1 and 2 were consistent with feedforward activation from local motion detectors providing a graduated input to the processing level at which motion patterns are formed: When the BRLC during the next-to-last display cycle was 0 , pattern-level hysteresis was eliminated or reversed. When it was 0.8 , there was a reliable hysteresis effect, and when it was 2.0 , hysteresis increased.

In this experiment, the BRLC was reduced from 0.8 to 0.4 during the display cycle with the perturbation. In previous research (Hock et al., 1997), motion and nonmotion were perceived approximately equally often for this BRLC value, so it was anticipated that during some trials there would be local decisions for motion perception to continue through the perturbation, but during other trials with the same stimulus there would be local decisions for nonmotion to be perceived instead. It was hypothesized that the local bistability resulting from this perturbation would determine whether or not there would be a brief interruption in the activation that feeds forward to patternlevel processing, thereby affecting pattern-level hysteresis. That is, different hysteresis effects were anticipated for the same stimulus, depending on whether motion or nonmotion was perceived during the perturbation.

The higher luminance value of each element during the perturbation was $7.1 \mathrm{~cd} / \mathrm{m}^{2}$, and the lower value was $6.3 \mathrm{~cd} / \mathrm{m}^{2}$. Examples of ascending trials with and without perturbations for 3 of the 4 participants (A.P., H.H., and
C.P.) were as described in Experiment 1; potential effects of motion adaptation were balanced in the perturbation and no-perturbation conditions when nonmotion was perceived during the BRLC $=0.4$ perturbation (see note 1 ). For the 4th participant (D.P.), trials with and without perturbations were as described in Experiment 2; potential adaptation effects for this participant would have been balanced in the perturbation and no-perturbation conditions, had motion been perceived during the $\mathrm{BRLC}=0.4$ perturbation (see note 2). A.P. and H.H. participated in six testing sessions, and C.P. and D.P. participated in four.

\section{Results}

The proportions of trials for which nonmotion was reported are presented for A.P. and H.H. in Figure 4. Although the BRLC of 0.8 strongly favored the perception of motion, the perception of nonmotion was reported during a small percentage of the trials in the noperturbation condition. This was attributable to occasional random fluctuations in activation that were large enough to result in the predominance of detectors responsive to motion-independent stimulus information, despite the stimulus strongly favoring detectors responsive to motionspecifying stimulus information (Hock et al., 1997). Significantly, the frequency of trials during which nonmotion was perceived was greater in the perturbation than in the no-perturbation condition. The additional nonmotion per-
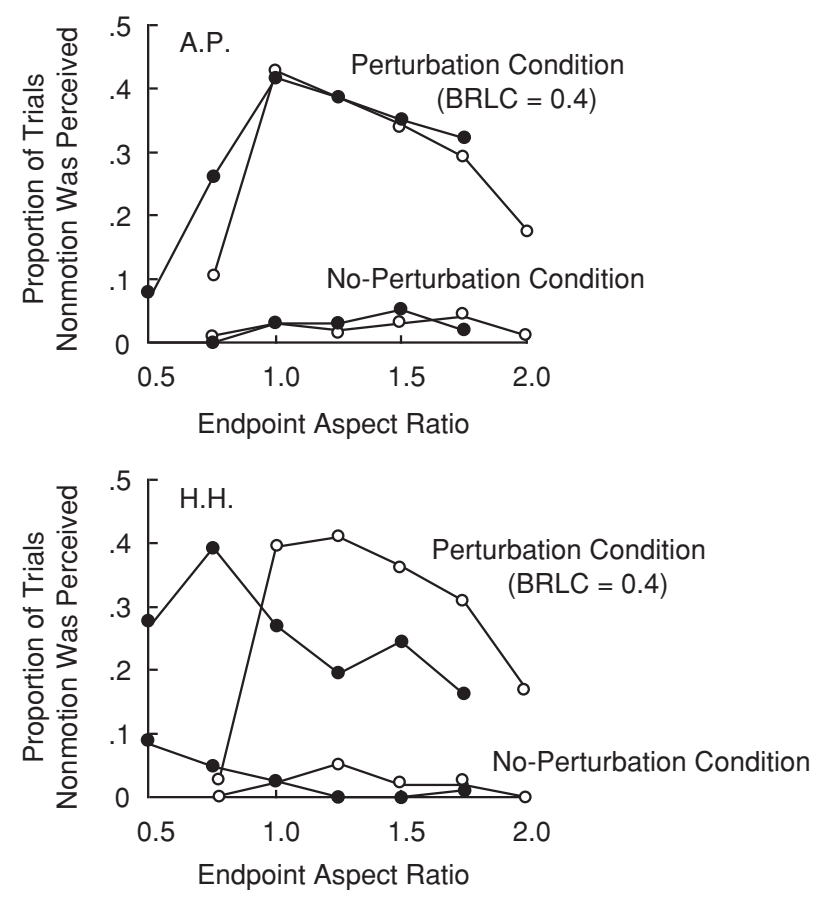

Figure 4. Experiment 3: Proportions of trials in which nonmotion was perceived by A.P. or H.H. sometime during the trial, presented separately for ascending trials (those with increasing aspect ratios; open circles) and descending trials (those with decreasing aspect ratios; filled circles). 


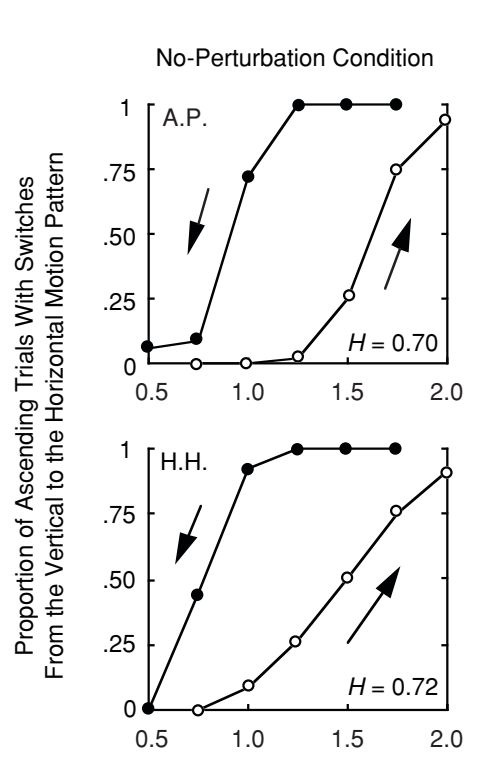
Perturbation $(B R L C=0.4)$ Condition: Perturbation $(B R L C=0.4)$ Condition: Motion Is Perceived for Entire Trial Nonmotion Is Perceived
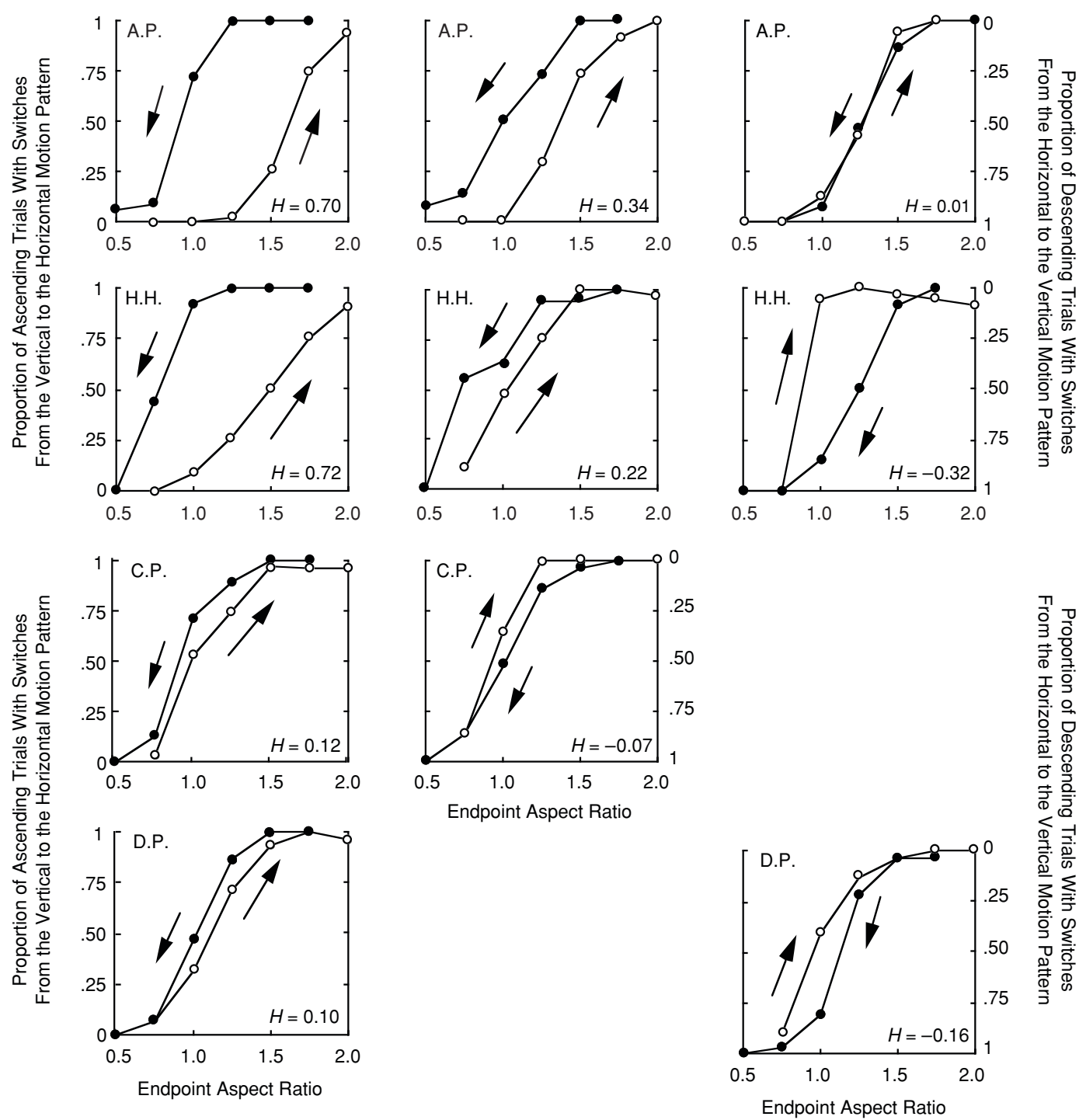

Figure 5. Experiment 3: Hysteresis effects for each participant in the no-perturbation and perturbation conditions (BRLC decreased from 0.8 to 0.4 during the next-to-last display cycle). The axes on the left side of each graph indicate the results for ascending trials (those with increasing aspect ratios; open circles). The results indicate the proportions of trials in which change to an alternative percept occurred sometime during each trial. The axes on the right side of each graph (note their inversion) indicate the results for descending trials (those with decreasing aspect ratios; filled circles). Trials in the perturbation condition are analyzed separately for A.P. and H.H., depending on whether motion or nonmotion was perceived during the perturbation. C.P. always perceived motion, and D.P. always perceived nonmotion during the perturbation interval. The size of each hysteresis effect, $H$, is indicated within each graph (see the text for the measurement procedure).

cepts when compared with the no-perturbation condition were attributable to the more balanced motion/nonmotion competition produced by the perturbation.

Perturbation trials in which nonmotion was perceived sometime during the trial were analyzed separately from those in which motion was perceived throughout. The results for A.P. and H.H. indicate that the outcome of local motion/nonmotion decisions strongly affected their pattern-level hysteresis (Figure 5), which was either eliminated (A.P.) or reversed (H.H.) when nonmotion was perceived. When motion continued to be perceived despite the perturbation, hysteresis continued to be observed for both A.P. and H.H. Motion/nonmotion bistability in the perturbation condition was minimal for the other 2 participants in the experiment. Motion almost always was perceived by C.P. despite the perturbation, and nonmotion always was perceived by D.P. as a result of the perturbation. Their hysteresis effects nonetheless were reduced in 
the perturbation condition relative to the no-perturbation condition (Figure 5), consistent with the reduction in feedforward activation when the BRLC was reduced from 0.8 to 0.4 during the perturbation.

\section{DISCUSSION}

The results of these three experiments indicate that the stability of the pattern perceived for the motion quartet (and thus, the size of pattern-level hysteresis) depends on the magnitude of feedforward activation from local motion detectors to pattern-forming mechanisms during the perturbation. This was the case even though the effect of the BRLC change during the perturbation was nonspecific with respect to the local activation of horizontal and vertical motion detectors (i.e., neither motion direction was differentially favored by the BRLC change). In Experiment 1 , a perturbation that reduced the BRLC from 0.8 to 0 strongly reduced and even reversed hysteresis. There was no motion-specifying stimulus information during this perturbation, so there was no feedforward flow of activation from local motion detectors to patternforming mechanisms. The opposite results were obtained in Experiment 2. A perturbation that increased the BRLC from 0.8 to 2.0 increased hysteresis. There was increased stimulus-initiated activation of local motion detectors during the perturbation, so the feedforward activation to the pattern level would have been increased. In addition to these graduated effects of stimulus-initiated activation due to local perturbations, the results of Experiment 3 indicated that pattern-level hysteresis depended on the outcome of the local motion/nonmotion competition caused by a perturbation that decreased the BRLC from 0.8 to 0.4 . When motion prevailed in the competition, hysteresis was obtained. When nonmotion was perceived as a result of the same perturbation, the stimulus-initiated activation of local motion detectors was suppressed by detectors responsive to motion-independent stimulus information; there would have been no feedforward activation from local motion detectors to pattern formation mechanisms, and hysteresis was eliminated or reversed.

The evidence thus indicates that local-level dynamical decisions (whether motion or nonmotion is perceived) and pattern-level decisions (whether the horizontal or vertical motion pattern is perceived) are linked through the magnitude of the activation that feeds forward from local detection to global pattern formation. This is because the future-shaping inhibitory interactions that are the basis for the stability of pattern-level decisions are activation dependent. The greater the feedforward flow of activation, the more strongly currently activated detectors inhibit soon-to-be-stimulated, competing motion directions (Hock et al., 2003). Our evidence that the level of feedforward activation affects pattern stability therefore supports the hypothesized activation dependence of these future-shaping interactions. The computational plausibility of this conclusion is supported by the dynamical simulations presented in the article that follows (Nichols et al., 2006).

\section{REFERENCES}

Bartsch, A., \& VAN Hemmen, J. L. (1997). A neural model of stroboscopic alternative motion. Network, 8, 107-126.

BiedERMAN, I. (1987). Recognition-by-components: A theory of human image understanding. Psychological Review, 94, 115-147.

CARMESIn, H. O., \& ARNDT, S. (1996). A neural network model for stroboscopic alternative motion. Biological Cybernetics, 75, 239-251.

CutTing, J. (1986). Perception with an eye for motion. Cambridge, MA: MIT Press.

Gilroy, L. A., Hock, H. S., \& Ploeger, A. (2001). Differential activation solution to the motion correspondence problem. Perception \& Psychophysics, 63, 847-861.

Hochstein, S., \& AhissaR, M. (2002). View from the top: Hierarchies and reverse hierarchies in the visual system. Neuron, 36, 791-804.

Hock, H. S., Kelso, J. A. S., \& SchöNER, G. (1993). Bistability and hysteresis in the organization of apparent motion patterns. Journal of Experimental Psychology: Human Perception \& Performance, 19, 63-80.

Hock, H. S., Kogan, K., \& Espinoza, J. K. (1997). Dynamic, statedependent thresholds for the perception of single-element apparent motion: Bistability from local cooperativity. Perception \& Psychophysics, 59, 1077-1088.

HocK, H. S., NichOLS, D. F., \& EsPINOZA, J. [K.] (2004). When motion is not perceived: Evidence from adaptation and dynamical stability. Spatial Vision, 17, 235-248.

Hock, H. S., SchöNER, G., \& Giese, M. (2003). The dynamical foundations of motion pattern formation: Stability, selective adaptation, and perceptual continuity. Perception \& Psychophysics, 65, 429-457.

HoEth, F. (1968). Bevorzugte Richtungen bei stroboskopischen Alternativbewegungen [Favored directions in stroboscopic alternative movements]. Psychologische Beiträge, 16, 494-527.

Hubel, D. H., \& Wiesel, T. N. (1979). Brain mechanisms of vision. Scientific American, 241, 150-162.

Kruse, P., Stadler, M., \& Wehner, T. (1986). Direction and frequency specific processing in the perception of long-range apparent movement. Vision Research, 26, 327-335.

Maunsell, J. H., \& Newsome, W. T. (1987). Visual processing in monkey striate cortex. Annual Review of Neuroscience, 10, 363-401.

Maunsell, J. H., \& Van Essen, D. C. (1983). Functional properties of neurons in middle temporal visual area of the macaque monkey: I. Selectivity for stimulus direction, speed, and orientation. Journal of Neurophysiology, 49, 1127-1147.

Movshon, J. A., \& Newsome, W. T. (1996). Visual response properties of striate cortical neurons projecting to area MT in macaque monkeys. Journal of Neuroscience, 16, 7733-7741.

Movshon, J. A., Thompson, I. D., \& Tolhurst, D. J. (1978). Receptive field organization of complex cells in the cat's striate cortex. Journal of Physiology, 283, 79-99.

Nichols, D. F., Hock, H. S., \& SCHÖNER, G. (2006). Linking dynamical perceptual decisions at different levels of description in motion pattern formation: Computational simulations. Perception \& Psychophysics, 68, 515-533.

Ramachandran, V. S., \& Anstis, S. M. (1985). Perceptual organization in multistable apparent motion. Perception, 14, 135-143.

SANOCKI, T. (1993). Time course of object identification: Evidence for a global-to-local contingency. Journal of Experimental Psychology: Human Perception \& Performance, 19, 878-898.

Snowden, R. J., Treue, S., Erickson, R. G., \& Andersen, R. A. (1991). The response of area MT and V1 neurons to transparent motion. Journal of Neuroscience, 11, 2768-2785.

Stoner, G. R., \& Albright, T. D. (1992). Neural correlates of perceptual motion coherence. Nature, 358, 412-414.

Thiele, A., Dobkins, K. R., \& Albright, T. D. (2000). Neural correlates of contrast detection at threshold. Neuron, 26, 715-724.

Vogels, R., \& Orban, G. A. (1996). Coding of stimulus invariances by inferior temporal neurons. Progress in Brain Research, 112, 195211

VON SChILler, P. (1933). Stroboskopische Alternativbewegungen [Stroboscopic alternative movements]. Psychologische Forschung, 17, 179-214.

Williams, D., Phillips, G., \& Sekuler, R. (1986). Hysteresis in the 
perception of motion direction as evidence for neural cooperativity. Nature, 324, 253-255.

Wilson, H. R., \& Kim, J. (1994). A model for motion coherence and transparency. Visual Neuroscience, 11, 1205-1220.

\section{NOTES}

1. Trials in the perturbation condition of Experiment 1 were longer by one display cycle than were trials with corresponding endpoint aspect ratios in the no-perturbation condition. The extra display cycle in the perturbation condition repeated the next-to-last aspect ratio, but with the BRLC reduced to 0 . Motion never was perceived during this display cycle, so the next-to-last aspect ratio was not repeated in the no-perturbation condition in order to equate the two conditions with respect to the number of frames during which motion was perceived (and thereby balance potential effects of motion adaptation).

2. Trials in the perturbation condition of Experiment 2 had the same duration as trials with corresponding endpoint aspect ratios in the no-perturbation condition. The next-to-last aspect ratio was repeated in both conditions. Motion always was perceived during the display cycle with the BRLC $=2.0$ perturbation, so the repetition of the next-to-last display cycle in the no-perturbation condition equated the two conditions with respect to the number of frames that motion was perceived (and thus balanced potential effects of motion adaptation).

(Manuscript received November 17, 2004; revision accepted for publication July 1, 2005.) 